Apuntes Universitarios, 2022: 12 (1), enero-marzo ISSN: 2304-0335 https://doi.org/10.17162/au.v11i5.919

\title{
Determinantes de la satisfacción laboral en un establecimiento de salud durante el estado de emergencia sanitaria, Perú
}

\author{
Determinants of job satisfaction in a health facility during the \\ state of health emergency, Peru \\ Edmer Mendoza-Briceño, ${ }^{1}$ María Auxiliadora Guerrero Bejarano, ${ }^{2 a}$ \\ Irma Carhuancho-Mendoza, ${ }^{3}$ Fernando Nolazco-Labajos, ${ }^{4}$ \\ Daniel Silva Siu ${ }^{5}$ \\ Ministerio de Salud-Lima Norte, Lima, Perú ${ }^{1}$ \\ Universidad Internacional del Ecuador, Guayaquil, Ecuador; Universidad Señor de \\ Sipán, Trujillo, Perú ${ }^{2}$ \\ Universidad Norbert Wiener; Universidad Privada César Vallejo, Lima, Perú ${ }^{3}$ \\ Universidad Privada César Vallejo, Lima, Perú ${ }^{4}$ \\ Universidad César Vallejo, Lima, Perú ${ }^{5}$ \\ (iD) ORCID ID: https://orcid.org/0000-0002-7452-737X ${ }^{1}$ \\ iD ORCID ID: https://orcid.org/0000-0002-1412-58702 \\ iD ORCID ID: https://orcid.org/0000-0002-4060-5667 3 \\ (iD) ORCID ID: https://orcid.org/0000-0001-8910-222X \\ (iD) ORCID ID: https://orcid.org/0000-0003-1783-6261
}

Recibido: 11 de mayo de 2021

Aceptado: 09 de setiembre de 2021

\section{Resumen}

En la presente investigación se examinaron los factores determinantes de la satisfacción laboral en un establecimiento de salud durante el estado de emergencia sanitaria en Perú. Se realizaron encuestas a 244 colaboradores de las diferentes áreas del establecimiento de salud. El estudio se realizó bajo el enfoque cuantitativo y de diseño ex post facto. La técnica de recopilación de datos fue la encuesta, la que se aplicó en el mes de agosto del 2020, cuando los casos de Covid 19 iban en aumento. Para la contrastación de la hipótesis se aplicó la regresión jerárquica por pasos, donde se determinó dos modelos que expliquen la satisfacción laboral. El primer modelo explica que la satisfacción laboral depende del burnout en un $20.4 \%$, con una potencia de 0.996 ; mientras que el segundo modelo explica la satisfacción laboral por el burnout y la motivación en un $28.7 \%$, con una potencia de 0.99 . Por lo tanto, en la investigación se confirmó que las determinantes de la satisfacción laboral son el burnout y la motivación según los colaboradores del establecimiento de salud durante el estado de emergencia sanitaria.

Palabras clave: Burnout, Covid 19, motivación laboral, salud, satisfacción laboral.

${ }^{\mathrm{a}}$ Correspondencia al autor:

E-mail: maguerrerobe@uide.edu.ec 


\begin{abstract}
In this research, the determining factors of job satisfaction in a health facility during the state of health emergency in Peru were examined. Surveys were conducted with 244 collaborators from the different areas of the health facility. The study was carried out under the quantitative approach and ex post facto design. The data collection technique was the survey, which was applied in August 2020, when Covid 19 cases were on the rise. Hierarchical stepwise regression was applied to test the hypothesis, where two models were determined to explain job satisfaction. The first model explains that job satisfaction depends on burnout in $20.4 \%$, with a power of 0.996 ; while the second model explains job satisfaction due to burnout and motivation in $28.7 \%$, with a power of 0.99 . Therefore, the research confirmed that the determinants of job satisfaction are burnout and motivation according to the collaborators of the health establishment during the state of health emergency.
\end{abstract}

Keywords: Burnout, Covid 19, job motivation, health, job satisfaction.

\title{
Introducción
}

El año 2020 ha sido atípico a nivel mundial por la presencia del Covid 19, lo cual obligó a que los países declaren el estado de emergencia sanitaria. Según la evidencia, este virus se originó en el continente asiático, en la Región de Hubei en China. Cabe precisar que, en el espacio de los primeros meses del año 2020, el virus se propagó por casi todos los países del mundo, siendo catalogado por las autoridades de salud mundial como una pandemia (Ruiz, 2020). La preocupación e incertidumbre, como característica concreta al desconocimiento del alcance de la enfermedad en la salud humana, ha conducido a tomar medidas preventivas ante esta emergencia; lo que originó que el área de salud fuera considerada como primera línea de contención de la enfermedad y por ello se definieron una serie de medidas de salubridad y protocolos para hacer frente a la pandemia; tanto en infraestructura como con utensilios de uso médico para que los profesionales de salud puedan desarrollar su actividad profesional en las entidades médicas, de tal forma que se reduzca el contagio o probabilidad de muerte (Fernández \& Ribeiro, 2020).

Ante esta pandemia, no solo es importante el paciente, sino también el personal médico que está expuesto al contagio y efectos colaterales, lo cual limita su desempeño laboral (Ribot, Chang \& González, 2020), altera el nivel de motivación, se deteriora la satisfacción laboral y eleva el nivel burnout por la incertidumbre que se vive (Polo et al., 2016). Frente a la realidad descrita se plantea la siguiente pregunta de investigación ¿Cuáles son las determinantes de la satisfacción laboral en un establecimiento de Salud durante el estado de emergencia sanitaria, Perú? 
Diferentes estudios previos han abordado la problemática mundial de la emergencia sanitaria, exponiendo los efectos de esta en la salud mental y el comportamiento organizacional en distintos países como Malasia (Plascencia et al., 2016), México y Perú. Los trabajos concluyen que existe un impacto significativo en la economía a consecuencia de las decisiones tomadas para afrontar la emergencia sanitaria (Duche-Pérez \& Rivera, 2019) y particularmente el Covid 19 (Huarcaya-Victoria, 2020). Esta situación ha generado la disminución de los sueldos, pero las horas de trabajo por el contrario se vieron incrementadas; teniendo como consecuencia problemas de cansancio, estrés, agotamiento mental y depresión (Mesfin et al., 2020). Sin embargo, los trabajadores de salud, educación y seguridad entre otros, durante la pandemia no han reducido sus jornadas de trabajo, que fueron incrementadas, para atender la demanda creciente en los mosocomios (Liza \& Shiang Lim, 2020).

\section{Revisión de la literatura}

La satisfacción laboral es definida como el estado emocional y actitud de los trabajadores en relación con sus funciones y puesto de trabajo (Gyamfi, 2014; Robbins et. al., 2013), evidenciándose en los resultados de la organización; además de clientes o usuarios satisfechos y el retorno de la inversión (Guerrero et. al., 2021; y Bejarano \& Siu, 2017). Estudios a partir de Vroom (1982) evidenciaron que un eje fundamental para lograr la satisfacción son las condiciones de trabajo (Ali, 2016).

En el caso de los establecimientos de salud, Holguín y Contreras (2020) indican que satisfacción laboral es un estadio emocional, que lleva al personal sanitario a ejercer sus actividades con autonomía, esperando el logro con eficiencia y eficacia. Sin embargo, durante la pandemia la carga laboral se incrementó, además de la tensión constante por el desconocimiento para atender a los pacientes (Arco \& Castillo, 2020) y el riesgo del contagio entre médicos y pacientes (Fernández \& Ribeiro, 2020).

Por otro lado, el género, la edad y otros factores pueden influir en la satisfacción de los empleados (El Badawy \& Magdy, 2015), en las personas de mayor edad está justificada en la responsabilidad, mientras que, para las mujeres es el salario (Saner \& Sadikoglu, 2016). Otros factores son: la raza (Zahari \& Shurbagi, 2012), la extroversión y escrupulosidad (Bandura \& Lyons, 2014; Yildirim, Gulmez, \& Yildirim, 2016), la esperanza, la autosuficiencia, la capacidad de resistencia y el optimismo (capital sicológico) (Jung \& Yoon, 2015). Los factores de motivación que influyen en la satisfacción laboral son: el reconocimiento, las metas alcanzadas, el desarrollo dentro del 
trabajo, y el sentido de justicia dentro de las organizaciones (López-Cabarcos et. al., 2015).

El burnout o síndrome del quemado es una de las consecuencias que suele surgir a partir de las condiciones de trabajo, que afectan de forma permanente o temporal, generando desgaste y disminución en la productividad (Maslach \& Jackson, 1981; Arco \& Castillo, 2020). Asimismo, toda actividad de servicio y atención al público son proclives de experimentar este síndrome (Marín \& Placencia, 2017).

La motivación comprende el conjunto de fortalezas predispuestas que influyen en el trabajo y en el trabajador, para cohesionar un comportamiento determinado de su accionar funcional, además para satisfacer con beneplácito sus expectativas y necesidades mediáticas (García \& Forero, 2014; Soto 2018).

\section{Método}

El enfoque del estudio fue cuantitativo, de nivel explicativo, y de diseño ex post facto. El método fue hipotético deductivo (Carhuancho et. al., 2019), a razón que la problemática observada fue durante el estado de emergencia sanitaria por el Covid 19, la medición de las variables se realizó con números a través de la escala ordinal.

Para el estudio participaron 150 mujeres y 94 varones que laboraban en los establecimientos de salud de la Diris Lima-Norte y que fueron informados sobre la realización del presente estudio. La muestra estuvo conformada por personal médico, enfermeras, técnicos de enfermería, tecnólogos especializados de laboratorio, fisioterapias, cirujanos dentistas, asistentas sociales, nutricionistas y obstetras. La edad estuvo entre 19 y 69 años, el personal tenía en promedio 17 años de servicio.

Los cuestionarios de las variables se distribuyeron a través del link generado en Google Forms, durante el mes de agosto del 2020. Se asumió que todos los participantes contestaron las preguntas verazmente y participaron voluntariamente del estudio una vez fueron informados del mismo. En la presentación del instrumento se incluyó el consentimiento informado donde el participante luego de la lectura aceptó contestar las preguntas, y que los resultados serían parte de una investigación que se publicaría en una revista indexada.

La primera parte del cuestionario contenía preguntas de información demográfica como género, área de trabajo, edad y tiempo de servicio en el establecimiento de salud. La siguiente sección estuvo conformada por el cuestionario de satisfacción laboral constituido por las dimensiones: a) significación de tareas; b) condiciones de trabajo; c) 
relaciones sociales; d) beneficios económicos; e) políticas administrativas; f) relaciones con la autoridad: g) desarrollo personal, comprendido por 36 ítems, cuya autora es Palma (2005). También se incluyó el cuestionario de Burnout, integrado por las dimensiones: a) agotamiento emocional; b) despersonalización; c) realización personal, con un total de 22 ítems, construido por Maslach \& Jackson (1986). Finalmente, el tercer cuestionario correspondió a la motivación, integrada por las dimensiones: a) desmotivación; b) regulación externa; c) regulación introyectada; d) regulación identificada; e) motivación intrínseca, haciendo un total de 19 ítems, los autores son Gagné, y otros (2010).

Los tres instrumentos se sometieron a la prueba de confiabilidad de Alfa de Cronbach, cuyos valores fueron superiores a $0.80(0.86,0.91,0.88)$, lo que indica una fuerte confiabilidad. Asimismo, los cuestionarios se sometieron a la validez de contenido por tres expertos, un doctor en psicología, un doctor en administración con especialización en recursos humanos, y un metodólogo-estadístico, para evaluar la consistencia de los ítems. Cabe precisar que, además tenían experiencia en la gestión hospitalaria y administrativa. Luego de la segunda revisión minuciosa suscribieron que los ítems respondían a las dimensiones, y ellas a la variable, por lo tanto, con la venia respectiva se procedió a la aplicación en la muestra de estudio (Carhuancho et. al., 2019). Para la contrastación de la hipótesis se aplicó la regresión jerárquica para determinar el modelo que explique el comportamiento de la satisfacción laboral a partir del burnout, y la motivación laboral, bajo el contexto del estado de emergencia sanitaria en el Perú.

Finalmente, en el desarrollo del trabajo se ha respetado y reconocido la autoría de los diferentes conceptos y publicaciones, donde se ha utilizado la norma de redacción APA, tanto para las citas y referencias. También se respetó cada una de las respuestas obtenidas, razón por la cual no se alteró o modificó registro alguno. En consecuencia, los resultados obtenidos y la contrastación de las hipótesis evidencian la realidad de estudio respecto a las variables que determinan la satisfacción laboral.

\section{Resultados}

En la tabla 1 se presentan las correlaciones positivas y significativas entre las variables, es así que la satisfacción laboral se correlaciona con el burnout en .452, y con la motivación .407, el nivel de significancia calculado fue menor a 0.05 . De los valores de correlación se desprende que cuanto mayor es el burnout y la motivación, mayor será la satisfacción laboral. 


\section{Tabla 1}

Correlaciones entre las variables de estudio

\begin{tabular}{llccc}
\hline & & $\begin{array}{c}\text { Satisfacción } \\
\text { laboral }\end{array}$ & Burnout & Motivación \\
\hline Correlación de & Satisfacción & 1,000 &, 452 &, 407 \\
Pearson & laboral & & & \\
& Burnout &, 452 & 1,000 &, 293 \\
& Motivación &, 407 &, 293 & 1,000 \\
Sig. (unilateral) $)$ & N_SL & $\cdot$ &, 000 &, 000 \\
& N_B &, 000 & $\cdot$ &, 000 \\
& N_M &, 000 &, 000 &. \\
\hline
\end{tabular}

Para el estudio se planteó la siguiente hipótesis: existen variables que determinan la satisfacción laboral en un establecimiento de salud durante el estado de emergencia sanitaria, Perú. Por tal motivo se aplicó la prueba de regresión jerárquica por pasos como se aprecia en la tabla 2 , donde se calculó el valor de R cuadrado, R cuadrado ajustado, la Beta, el error estándar, el nivel de significancia, la potencia estadística y el tamaño del efecto. Es así que se estructuró dos modelos que explican la satisfacción laboral, el modelo 1 predice o explica la satisfacción laboral en $20.4 \%$, además que la potencia del modelo es del .996; mientras que el modelo 2 explica la satisfacción laboral a través del burnout y la satisfacción laboral en $28.7 \%$ con la potencia de $99 \%$. La ecuación que se estructura para cada modelo es: a) Modelo 1: $\mathrm{Y}_{1}=1.057+0.417 * \mathrm{~B}$; y b) Modelo 2: $\mathrm{Y}_{2}=$ $.682+0.336 * \mathrm{~B}+.286 * \mathrm{M}$. 


\section{Tabla 2}

Modelos que explican la satisfacción laboral

\begin{tabular}{lcccccccccc}
\hline Modelos & $\mathrm{F}$ & $\mathrm{R} 2$ & $\Delta \mathrm{R} 2$ & $\mathrm{~B}$ & $\begin{array}{c}\text { Error } \\
\text { estándar }\end{array}$ & $B$ & $\mathrm{p}$ & $1-\beta$ & $f^{2}$ \\
\hline Modelo 1 & 62.07 & .204 & .201 & .417 & .053 & .452 & .001 & .996 & .2562 \\
Burnout & $(1,242)$ & & & & & & & & \\
Modelo 2 & 48.486 & .287 & .281 & & & & .001 & .990 & .3908 \\
Burnout & $(2,241)$ & & & .336 & .052 & .364 & & & \\
Motivación laboral & & & & .286 & .054 & .301 & & & \\
\hline
\end{tabular}

Asimismo, se calculó el valor VIF para cada variable independiente, para el modelo 1 el valor calculado fue de 1.000, y en el modelo 2 correspondió a 1.094, lo cual demuestra que efectivamente las variables determinadas son independientes. También se calculó el valor de Durbin y Watson de 1.857, lo cual evidencia que existe autocorrelación entre las variables determinadas para los modelos.

\section{Discusión}

Los resultados del estudio apoyan lo sostenido en la teoría de higiene motivacional, porque es importante para el desempeño laboral del personal de salud las condiciones como infraestructura, la disponibilidad de agua potable, instrumental médico, los equipos de protección personal como son las mascarillas, los respiradores, las batas, los guantes quirúrgicos, entre otros que los proteja del contagio del Covid 19 (Ali, 2016). También es significativo valorar la teoría de la expectativa de Vroom (1982) porque el gobierno de turno ofreció reconocer con sueldos por encima del promedio al personal que se incorporaba para atender a los pacientes por el virus antes mencionado; ello generó expectativa en muchos jóvenes, quienes exponiendo su salud, a cambio de un sueldo diferenciado se enrumbaron a las ciudades donde la estadística de contagio y muerte era elevada como Lima, Iquitos y Piura.

Sin embargo, es importante enfatizar que las jornadas laborales eran superior a las 8 horas, el personal tenía que adquirir sus propios equipos de protección, existieron varios casos de contagio entre el personal, y la estadística de muerte fue elevada, todo ello ocasionó que el nivel de burnout se elevará, pero que a la vez la motivación del equipo humano por salvar vidas y atender a cada paciente era elevada, tal es así que se observó bastante involucramiento del personal, exponiendo a sus familias al contagio. 
Los resultados revaloran lo expuesto por Maslach y Jackson (1981), porque el personal de salud está expuesto al cansancio y desgaste emocional por las jornadas extenuantes que tienen que enfrentar para atender a los pacientes con Covid 19, lo cual conlleva a mermar los niveles de satisfacción laboral. En la investigación se demostró que el burnout y la motivación laboral influyen en la satisfacción laboral, el modelo 2 explica en un $28.7 \%$ el comportamiento de la variable dependiente, esto quiere decir que a pesar que el personal trabaje bajo presión y jornadas amplias, con escasos recursos para la atención al paciente y exposición al riesgo, se encuentran satisfechos con la labor loable que despliegan en la atención a los pacientes con Covid 19, esto realza el compromiso que adquirieron al momento de optar por una formación profesional vinculada a la salud, donde prima el prójimo, a pesar de exponer su propia vida.

El resultado del estudio concuerda con Duche-Pérez y Rivera (2019) porque el actuar y desenvolvimiento de las personas responde a los objetivos planteados, que para el caso correspondió el salvar vidas por el Covid 19. En este mismo sentido se concuerda con Mesfin et. al. (2020) porque menos del 30\% se encontraban satisfechos con el espacio físico del hospital, este problema es común en los diferentes establecimientos de salud en el mundo, peor aún fue en esta época porque se tuvieron que improvisar espacios para triaje y hospitalización, exponiendo a la adquisición de otras bacterias y virus porque el lugar no tenía las condiciones mínimas necesarias.

De manera similar, se concuerda con el estudio de Huarcaya-Victoria (2020) quién expuso sobre el deterioro emocional de las personas por el aislamiento social, lo cual se viene evidenciando a través de problemas familiares, violencia física y psicológica, que también ataña al personal de salud, en este mismo contexto se viene evidenciando casos de depresión por la pérdida de familiares, lo cual genera a la vez el agotamiento emocional y desmotivación para seguir trabajando. También se coincide con Plascencia et. al. (2016) porque el personal de salud ante la situación crítica que se vive, se ha visto afectado en el ámbito emocional, por ello es importante que en el Plan de trabajo del 2021 se implementé programas para atender a dicho personal, además de reconocer y compensar las horas trabajadas en exceso.

Este resultado también se coincide con Liza y Shiang Lim (2020) porque la situación de la pandemia obligó a las personas entre elegir trabajar o no, considerando el riesgo de la salud, a pesar de ello, varios profesionales de salud se enrolaron a la causa de atender a los pacientes y aplicaron sus conocimientos para salvar las vidas. Este estudio estuvo limitado a la variable burnout en el contexto de la pandemia Covid-19 y en casas 
de salud del Perú; por lo que los resultados no podrían generalizarse a todo tipo de situaciones o empresas.

Finalmente, se sugiere que las próximas investigaciones aborden otras variables de estudio como la carga laboral, el empowerment, el clima organizacional para que se diseñen modelos que expliquen la satisfacción laboral del personal de salud ante la pandemia que se vive. Asimismo, el principal hallazgo del estudio corresponde a que prima en el equipo humano del sector salud el servicio por la comunidad, a pesar de exponer su propia vida, con el único fin que las personas recuperen el estado de salud; se recomienda aplicar los instrumentos a profesionales de otros establecimientos de salud para demostrar que el modelo 2 explica la satisfacción laboral. Finalmente, el estudio ha tenido cierta limitación en lo que respecta al alcance de un mayor número de colaboradores, porque ante la recarga laboral y el cansancio, varios colaboradores no completaron los instrumentos en el tiempo previsto, otros abandonaron el link luego del avance por considerar que existía demasiadas preguntas, ello conlleva a pensar en un futuro estudio para estandarizar el cuestionario bajo el contexto del estudio en el Perú.

En consecuencia, el estudio revela una situación real y vigente ante el confinamiento mundial, en el Perú el nivel de contagios ha disminuido de manera considerable, sin embargo, se proyecta una segunda ola, lo cual obligará al personal de salud a no doblegar sus esfuerzos por atender los próximos casos, por ende, motivará a una siguiente investigación y comparar los niveles de satisfacción laboral.

\section{Conclusión}

El estudio de las determinantes de la satisfacción laboral en un establecimiento de salud durante el estado de emergencia sanitaria, Perú, provee de evidencia para con los resultados explicar que el burnout y la motivación laboral influyen en la satisfacción según el personal del sector salud, que para el caso fue del $28.7 \%$, entonces existen otras variables que expliquen la variabilidad de los resultados, que ameritarían profundizar en el estudio respectivo.

La satisfacción está relacionada con la viabilidad de su accionar en un escenario acorde para tal fin. En el caso de los centros de atención sanitaria se debe brindar estas condiciones de manera integral. En situaciones apremiantes como riesgo y vulnerabilidad de los servicios médicos como los suscitados por la emergencia sanitaria deben adecuarse a los protocolos correspondientes y disponer del apoyo profesional para evitar la sobrecarga laboral y el deterioro de sus funciones cotidianas. En ese sentido, la conducta 
del trabajador es el reflejo de la reacción empática con su entorno. Así, la satisfacción es percibida como el beneplácito acontecido como resultado de la actividad funcional que desarrolla en su organización.

Por otra parte, la motivación laboral en los centros médicos y en situaciones apremiantes como la emergencia sanitaria por la pandemia se ha visto afectada a factores emergentes que van desde el desconocimiento de las características de la enfermedad como los síntomas y su inmunología en la población. Es importante considerar que la motivación y su predisposición efectiva hacia las acciones determinadas de una función de trabajo se encuentran relacionadas con elementos afines a las necesidades humanas, el incentivo o recompensa y la voluntad que impulsa su materialización. Por lo tanto, la motivación no solo es el motor efectivo que impulsa el accionar de los eventos humanos, sino que permite la pertinencia de los mismos para la consecución de actividades laborales frente a diversas situaciones como la emergencia sanitaria y la predisposición de trabajar de manera efectiva a pesar de las implicancias que puede acarrear la presencia de esta enfermedad contagiosa en el entorno de trabajo.

\section{Referencias}

Ali, W. (2016). Understanding the Concept of Job Satisfaction, Measurements, Theories and its Significance in the Recent Organizational Environment: A Theoretical Framework. Archives of Business Research, 4 (1), 100-111. https://journals.scholarpublishing.org/index.php/ABR/article/view/1735

Arco, L., \& Castillo, J. (2020). Síndrome de Burnout en época de pandemia: caso colombiano. Interconectando saberes, 5 (10), 115-123. DOI: https://doi.org/10.25009/is.v0i10.2675

Bandura, R. P., \& Lyons, P. R. (2014). The art and science of job satisfaction: Much of it comes down to decent management practice. Human Resource Management International Digest, 22 (7), 32-35. doi:doi:10.1108/HRMID-10-2014-0141

Bejarano, M. \& Siu, D. (2017). La lealtad de los clientes y su relación con la lealtad de los colaboradores. INNOVA Research Journal, 2 (9), 121-125. doi: https://doi.org/10.33890/innova.v2.n9.2017.477

Carhuancho-Mendoza, I., Sicheri, L., Nolazco, F., Guerrero, M. \& Casana, K. (2019). Metodología de la investigación holística. Guayaquil: UIDE.

Carrillo, C., Martínez, M. E., Gómez-García, C. I., \& Meseguer, M. (2015). Satisfacción laboral de los profesionales sanitarios de un hospital universitario: análisis general 
y categorías laborales. Anales de psicología, 31 (2), 645-650. Recuperado de https://revistas.um.es/analesps/article/view/analesps.31.2.169791/175731

Duche-Pérez, A. \& Rivera, G. (2019). Job satisfaction and happiness in Peruvian nurses. Enfermería Global, 18 (2) 364-373. https://doi.org/10.6018/eglobal.18.2.334741.

El Badawy, T. A., \& Magdy, M. M. (2015). Assessing the Impact of Emotional Intelligence on Job Satisfaction: An Empirical Study on Faculty Members with Respect to Gender and Age. International Business Research, 8 (3), p67. DOI:10.5539/ibr.v8n3p67

Fernández, M. A., \& Ribeiro, A. (2020). Salud mental y estrés ocupacional en trabajadores de la salud a la primera línea de la pandemia de COVID-19. Revista Cuidarte, 11 (2). https://doi.org/10.15649/cuidarte.1222

García, M. \& Forero, C. (2014). Motivación y satisfacción laboral como facilitadores del cambio organizacional: Una explicación desde las ecuaciones estructurales, $\begin{array}{llll}\text { Psicogente, } & 17 & \text { (31), }\end{array}$ https://dialnet.unirioja.es/servlet/articulo?codigo $=6113728$

Gyamfi, G. (2014). Influence of job stress on job satisfaction: Empirical evidence from ghana police service. International Business Research, 7 (9), p108. DOI:10.5539/ibr.v7n9p108

Guerrero, M., Manosalvas, C., Salvador, C. , Carhuancho-Mendoza, I. , Maino, A. , \& Silva, D. (2021). La mediación de la satisfacción laboral en la relación del estilo de liderazgo y el compromiso organizacional. Apuntes Universitarios, 11 (2), 234 - 265. https://doi.org/10.17162/au.v11i2.657

Holguín, Y., \& Contreras, C. (2020). Satisfacción laboral del personal en el Servicio de Nefrología del Hospital Guillermo Kaelin de la Fuente (Villa María) en 2019. Horizonte Médico (Lima), $\quad 20 \quad$ (2), $\quad$ e1238. https://dx.doi.org/10.24265/horizmed.2020.v20n2.09

Huarcaya-Victoria J. (2020). Consideraciones sobre la salud mental en la pandemia de COVID-19. Rev Peru Med Exp Salud Publica, 37 (2): 327-34. Doi: https://doi.org/10.17843/rpmesp.2020.372.5419

Jung, H. S., \& Yoon, H. H. (2016). What does work meaning to hospitality employees? The effects of meaningful work on employees' organizational commitment: The mediating role of job engagement. International Journal of Hospitality Management, 53, 59-68. https://doi.org/10.1016/j.ijhm.2015.12.004 
Liza, J., \& Shiang Lim, E. (2020). Characterisation of Workers with Full-Time and PartTime Work Schedules: The Labour Market in Penang, Malaysia. Pertanika J. Soc. $\begin{array}{llllllll}\text { Sci. } & \& & \text { Hum. } & 28 & \text { (3), } & 2389 & - & 2404 .\end{array}$ http://www.pertanika.upm.edu.my/Pertanika\%20PAPERS/JSSH\%20Vol.\%2028 \%20(3)\%20Sep.\%202020/45\%20JSSH-5284-2019.pdf.

López-Cabarcos, M. Á., Machado-Lopes-Sampaio-de Pinho, A. I., \& VázquezRodríguez, P. (2015). The influence of organizational justice and job satisfaction on organizational commitment in Portugal's hotel industry. Cornell Hospitality Quarterly, 56 (3), 258-272. https://doi.org/10.1177/1938965514545680

Marín, H., \& Placencia, M. (2017). Motivación y satisfacción laboral del personal de una organización de salud del sector privado, Horiz. Med, 17 (4), 42-52. DOI: https://doi.org/10.24265/horizmed. 2017.v17n4.08

Maslach, C. \& Jackson, S. E. (1981). MBI: Maslach Burnout Inventory Manual. California: Consulting Psychologists Press

Mesfin, D., Woldie, M., Adamu, A., \& Bekele, F. (2020). Perceived organizational culture and its relationship with job satisfaction in primary hospitals of Jimma zone and Jimma town administration, correlational study. BMC Health Services Research, 20 $1-9$. https://bmchealthservres.biomedcentral.com/articles/10.1186/s12913-02005319-x

Plascencia, A., Pozos, B., Preciado, M., \& Vázquez, J. (2016). Satisfacción laboral del personal de enfermería de una institución pública de Jalisco, México. Revista Cubana de Salud y $\quad$ Trabajo, $2 \quad$ (1), 42-46. https://www.medigraphic.com/pdfs/revcubsaltra/cst-2016/cst162g.pdf

Polo, J. D., Santiago, V., Navarro, M. C. \& Alí, A. (2016). Creencias irracionales, síndrome de Burnout y adicción al trabajo en las organizaciones. Psicogente, 19 (35), 148- 160. http://doi.org/10.17081/psico.19.35.1215

Ribot, V., Chang, N. \& González, L. (2020). Efectos de la COVID-19 en la salud mental de la población. Revista Habanera de Ciencias Médicas, 19, 1-11. http://www.revhabanera.sld.cu/index.php/rha b/article/view/3307/2531

Robbins, S., Judge, T. A., Millett, B., \& Boyle, M. (2013). Organizational behaviour: Pearson Higher Education AU.

Ruiz, M. (2020). Salud mental y Covid-19: consejos para combatir el aislamiento por la pandemia [Internet]. La Habana: Gaceta Medica [Citado 25/10/2020]. Disponible 
en http:/gacetamedica.com/profesion/saludmental-y-covid-19-consejos-paracombatir-elaislamiento-por-la-pandemia/

Saner, T., \& Sadikoglu, G. (2016). Gender differences in job satisfaction in 5-star hotels of north Cyprus: descriptive analysis. Procedia Computer Science, 102, 359-364. https://doi.org/10.1016/j.procs.2016.09.412

Soto, M. (2018). Estudio de la motivación laboral en el personal del Centro de Salud La Esperanza. Revista Médica, $11 \quad$ 27-31. https://revista.hospitaltacna.gob.pe/index.php/revista2018/article/view/60/44

Vroom, V. (1982). Work and motivation. Filadelfia: Krieger Publishing Company

Yang, Y., Li, W., Zhang, Q., Zhang, L., Cheung, T., \& Xiang, Y. T. (2020). Mental health services for older adults in China during the COVID-19 outbreak. Lancet Psychiatry, 7 (4): e19. doi: http://dx.doi.org/10.1016/s2215-0366(20)30079-1

Yildirim, B. I., Gulmez, M., \& Yildirim, F. (2016). The relationship between the fivefactor personality traits of workers and their job satisfaction: S study on five-star hotels in Alanya. Procedia Economics and Finance, 39, 284-291. doi:10.1016/S2212-5671(16)30325-2

Zahari, I. B., \& Shurbagi, A. M. A. (2012). The effect of organizational culture and the relationship between transformational leadership and job satisfaction in petroleum sector of libya. International Business Research, 5 (9), 89. DOI:10.5539/ibr.v5n9p89 